\title{
MicroRNA Expression Profiles Related to Early Stage Murine Concanavalin A-Induced Hepatitis
}

\author{
Hong-Yu Jia ${ }^{a, b}$ Feng Chen ${ }^{a, b}$ Jian-Zhong Chen ${ }^{c}$ Shan-Shan Wua,b Jing Wanga,b \\ Qing-Yi Cao ${ }^{a, b}$ Zhi Chen ${ }^{a, b}$ Hai-Hong Zhua,b \\ aState Key Laboratory for Diagnosis and Treatment of Infectious Diseases, Institute of Infectious \\ Diseases, First Affiliated Hospital, School of Medicine, Zhejiang University, ${ }^{b}$ Collaborative Innovation \\ Center for Diagnosis and Treatment of Infectious Diseases, Hangzhou, 'Institute of Immunology, School \\ of Medicine, Zhejiang University, Hangzhou, China
}

\section{Key Words}

Fulminant hepatitis $\cdot$ microRNAs $・$ Expression profile $\bullet$ Hepatic failure

\begin{abstract}
Background: Fulminant hepatitis is a severe liver disease characterized by massive hepatocyte necrosis and clinical signs of liver failure. This study explores the expression profile of microRNAs, which are regulators of a number of pathophysiological processes, during the early stage of concanavalin A (Con A)-induced hepatitis. Methods: Balb/c mice were given ConA injections to induce fulminant hepatitis. miRNA expression profiling in liver tissues was carried out by microarray analysis. The differentially expressed miRNAs were subjected to time sequence profile analysis, gene-miRNA regulatory network analysis, and gene ontology-miRNA regulatory network analysis. Results: Eleven miRNAs among multiClass were found to be significantly differentially expressed between liver tissue in early stage fulminant hepatitis and normal control liver tissue. Mmu-miR-133a was the most differentially expressed with the strongest regulatory ability, regulating 47 mRNAs. Mmu-miR-10a was the most highly expressed in the microRNA-GO-Network and also exerted a strong regulatory ability. The expression profiles of miR-133a and miR-10a were verified by RT-PCR. Conclusions: These results show that, in the early stage, ConA-induced fulminant hepatitis induces a distinct miRNA expression profile. This differential miRNA expression profile may provide pathogenic clues and potential diagnostic and prognostic markers in acute and severe liver disease.
\end{abstract}

H.-Y. Jia, F. Chen and J.-Z. Chen contributed equally to this work. 


\section{Cellular Physiology and Biochemistry}

Cell Physiol Biochem 2014;33:1933-1944

DOI: 10.1159/000362970

online: June 27, 2014

C 2014 S. Karger AG, Basel

www.karger.com/cpb

1934

\section{Introduction}

Liver disease is a major health care problem worldwide. More than 2 billion people are estimated to be infected with hepatitis viruses, and approximately $1 \%$ will develop acute fulminant hepatitis. Moreover, the liver is a target for autoreactive lymphocytes that can result in autoimmune hepatitis. Fulminant hepatitis is a severe acute liver dysfunction characterized by massive hepatocyte necrosis and by clinical features of liver failure, such as coagulation dysfunction, hepatic encephalopathy, and ascites. Severe hepatitis progresses rapidly, with poor prognosis and high mortality. The progression from mild to severe hepatitis is an exacerbation process, in which hepatic tissue undergoes rapid massive or submassive necrosis. In both viral and autoimmune hepatitis, macrophage and T-cell activation are critical initial steps in the pathogenesis of liver damage and fulminant liver disease [1], although the intimate molecular mechanisms responsible for this exacerbation remain unclear.

In mice, fulminant, $T$ cell-dependent hepatitis and acute liver damage can be induced by a single intravenous (i.v.) injection of concanavalin A (ConA) [2]. ConA, a widely used plant lectin, isolated from Canavalia ensiformis (Jack bean) seeds, can agglutinate cells by specifically binding surface mannose and glucose residues. ConA-induced hepatitis is both $\mathrm{T}$ cell- and macrophage-dependent, as it cannot be induced in nude athymic mice that lack immunocompetent $\mathrm{T}$ cells and is inhibited by T-cell immunosuppressive drugs such as cyclosporine A (CsA) and FK506. Murine ConA-induced hepatitis and human virus-induced hepatitis share several pathological features, including increased production of tumor necrosis factor- $\alpha$ (TNF- $\alpha$ ) and interferon- $\gamma$ (IFN- $\gamma$ ), liver cell apoptosis, and the involvement of cytotoxic T cells. As such, murine ConA-induced hepatitis is a useful model for studying viral and autoimmune hepatitis [3].

MicroRNAs (miRNAs) are a highly evolutionarily conserved class of small, noncoding RNAs that can play important regulatory roles in animals and plants by targeting mRNAs for cleavage or translational repression. They are approximately 21 to 22 nucleotides (nt) in length, cleaved from $\sim 70$-nt (in animals) or $~ 100$-nt (in plants) hairpin precursors with signature 3'-hydroxyl and 5'-monophosphate termini in an RNase III cleavage event that leaves a 2-nt overhang. The cleavage depends on the consecutive actions of at least two endonucleases. miRNAs act as important post-transcriptional regulators of gene expression by base-pairing to their target mRNAs and mediating mRNA cleavage or translational repression [4]. In animals, they block translation by imperfect base-pairing to the $3^{\prime}$-untranslated regions (3'-UTR) of specific mRNAs and by induction of mRNA degradation.

miRNAs are involved in a range of physiological responses including developmental timing and participate in the regulation of multiple processes including cell fate determination, proliferation, differentiation, and cell death. Increasing attention has been paid to the functions of miRNAs in disease states. It is clear that miRNAs regulate many aspects of the innate and acquired immune response. For example, miR-146, miR-155, and miR-223 are thought to regulate the acute inflammatory response after the recognition of pathogens by the Toll-like receptors (TLRs), whereas miR-155 and miR-181a are implicated in B- and T-cell responses [5]. Recent data suggest that miRNAs may play significant roles in fulminant hepatitis [6]. miR-221 may serve as a potent posttranscriptional regulator of FAS-induced apoptosis. Interference using adenovirus-mediated artificial miRNAs targeting mfgl2, mFas, and mTNFR1 might have significant therapeutic potential for the treatment of fulminant hepatitis [7]. An et al. reported that miR-15b and miR-16 regulate TNF-mediated hepatic apoptosis via BCL2 during acute liver failure (ALF) in the D-galactosamine (D-GalN) mouse model [8] .

Profiling miRNA expression during the development of hepatitis is important for studying the biological functions of miRNAs and gene regulation. Whole miRNA and gene expression analyses provide a system in which the crucial factors controlling injury to the liverfollowing a ConA injection, and the post-transcriptional mechanisms regulating the ConA-stimulated liver response, can be examined.Large-scale studies on miRNA expression 


\section{Cellular Physiology and Biochemistry}

Cell Physiol Biochem 2014;33:1933-1944

DOI: $10.1159 / 000362970$

Publisned online: June 27, 2014

Jia et al.: ConA-Induced Hepatitis MicroRNA Expression Profiles

profiles have been carried out in many model organisms using Northern blot analysis and miRNA cloning [3-8]. However, both methods are time consuming. Microarray analysis technology allows the expression of thousands of genes to be profiled simultaneously. These data prompted us to explore miRNA profiling as a potential pathogenic and diagnostic marker during the early stage of a murine ConA-induced model of severe hepatitis.

\section{Materials and Methods}

\section{Animals and treatment}

Male BALB/c mice (18-20 g) were obtained from the Animal Center of Zhejiang Academy of Medical Sciences. The study was approved by the animal ethics committee of Zhejiang University. All experiments were conducted according to the institutional rules of animal experimentation. ConA (Type IV, Sig), dissolved in $100 \mu \mathrm{l}$ pyrogen-free normal saline, was administered to the mice $(20 \mathrm{mg} / \mathrm{kg})$ via tail vein injection. Control mice were given saline alone. The applied histological and biological assays have been previously reported [9]. Three biological replicate samples of serum and liver tissue were collected at 0,1 , 3 , and $6 \mathrm{~h}$ after ConA injection $[3,9]$.

\section{RNA isolation and $\mu$ Paraflo ${ }^{T M}$ miRNAmicroarrayassay}

$\mu$ Paraflo ${ }^{\mathrm{TM}}$ microfluidic chips (LC Sciences) were used in this study. Total RNA was isolated from liver tissue using TRIzol (Invitrogen, Carlsbad, CA, USA) according to the provided protocol. The quantity and quality of the isolated RNA were assessed by the Agilent Bioanalyzer ${ }^{\mathrm{TM}}$ (Agilent Technologies, Foster City, CA, USA) and by spectrophotometer using $260 \mathrm{~nm} / 280 \mathrm{~nm}$ ratios and $260 \mathrm{~nm} / 230 \mathrm{~nm}$ ratios. The miRNA microarray assay was performed by LC Sciences (http://www.lcsciences.com; Houston, TX). Two to five micrograms of total RNA were size fractionated using a YM-100 Microcon centrifugal filter (Millipore) and the small RNAs ( $<300 \mathrm{nt})$ isolated were 3 '-extended with a poly(A) tail using poly(A) polymerase. An oligonucleotide tag was then ligated to the poly(A) tail for later fluorescent dye staining; two different tags were used for the two RNA samples in dual-sample experiments. Hybridization was performed overnight on a $\mu$ Paraflo ${ }^{\mathrm{TM}}$ microfluidic chip using a micro-circulation pump (Atactic Technologies). On the microfluidic chip, each detection probe consisted of a chemically modified nucleotide coding segment complementary to the target miRNA (from miRBase, http://microrna.sanger.ac.uk/sequences/) or other RNA (control or customer-defined sequences), and a spacer segment of polyethylene glycol to extend the coding segment away from the substrate. The detection probes were made by in situ synthesis using photogenerated reagent (PGR) chemistry. Hybridization melting temperatures were balanced by chemical modifications of the detection probes. After hybridization in $100 \mu \mathrm{L} 6 \times \mathrm{SSPE}$ buffer $\left(0.9 \mathrm{M} \mathrm{NaCl}, 60 \mathrm{mM} \mathrm{Na}_{2} \mathrm{HPO}_{4}, 6 \mathrm{mM}\right.$ EDTA, $\mathrm{pH}$ 6.8) containing $25 \%$ formamide at $34^{\circ} \mathrm{C}$, fluorescence labeling with tag-specific Cy5 dyes was used for detection. Images were collected using a laser scanner (GenePix 4000B microarray scanner, Molecular Device) and digitized using Array-Pro image analysis software (Media Cybernetics). Data were analyzed by first subtracting the background and then normalizing the signals using a LOWESS filter (locally-weighted regression).

\section{Affymetrix microarray analyses}

Total RNA was isolated as described above and purified using the RNeasy system (QIAGEN, Valencia, CA).The quantity and quality were assessed as described above. The RNA was purified using the QIAGEN RNeasy Total RNA Isolation kit (QIAGEN, GmbH, D-40724, Hilden, Germany) according to the manufacturer's protocol. Poly $(\mathrm{A})^{+} \mathrm{mRNA}$ was isolated using the QIAGEN Oligotex Direct mRNA kit. Gene expression microarray hybridizations were done using the GeneChip ${ }^{\circledR}$ Mouse Genome 4302.0 Array (Affymetrix) and performed by Shanghai Biochip Co., Ltd. RNA labeling and hybridization followed published methodologies [10]. The results were listed in another paper [9] and deposited in Gene Expression Omnibus (GEO; http://www.ncbi.nlm.nih.gov/projects/geo/), and the records have been approved and assigned GEO accession numbers (GSE17184) as can be found here:http://www.ncbi.nlm.nih.gov/geo/query/acc. cgi?acc $=$ GSE17184.

\section{Significant differential miRNA analysis among multiClass}

The sample datasets were obtained from miRNA microarray analyses at $0,1,3$, and $6 \mathrm{~h}$ after injection with $20 \mathrm{mg} / \mathrm{ml}$ ConA solution. Each group included three samples. Accurate estimation of miRNA signal 


\section{Cellular Physiology and Biochemistry}

Cell Physiol Biochem 2014;33:1933-1944

\begin{tabular}{l|l}
\hline DOI: $10.1159 / 000362970$ & (c) 2014 S. Karger AG, Basel
\end{tabular}

www.karger.com/cpb

Jia et al.: ConA-Induced Hepatitis MicroRNA Expression Profiles

variability was difficult, because the number of samples (3 per group) was far less than the number of potential miRNAs and there were few degrees of freedom in miRNA expression signal variance. Significant differential miRNA expression derived from comparison of the three samples was filtered by an analysis of variance (ANOVA)-corrected random variance model (RVM). The multiple comparison and permutation tests were repeated 1000 times to estimate the frequency of the false discovery rate (FDR) for assigning significance to differential miRNAs expression and the random chance of significance being assigned. The threshold for true significance in miRNA expression was $p<0.1$ and FDR $<0.05$, whereas the threshold for significant differential miRNA expression was $p<0.05$.

\section{Time sequence profile analysis of miRNA expression}

Differential miRNAs obtained at four time points were filtered by RVM ANOVA, and then sets of distinct model expression profiles were studied. For analytical purposes, it was assumed that the raw expression values were converted into $\log _{2}$ ratio. miRNA expression values from the four time points $(0,1,3$, and 6 h) were transformed to $\log _{2} \frac{0 h}{0 h}, \log _{2} \frac{1 h}{0 h}, \log _{2} \frac{6 h}{0 h}$, and $\log _{2} \frac{6 h}{0 h}$, respectively. In this experiment, 26 profiles were defined according to the cluster analysis strategy of short-time series experiments. Expression model profiles were defined by the number of miRNAs assigned to each model profile and the expected number. miRNA profiles were assessed for statistical significance by Fisher exact and multiple comparison tests. miRNA profiles at each time point were deemed significant at a $p<0.05$.

\section{Construction of gene-miRNA regulatory network and topological analysis}

The gene-miRNA regulatory network was constructed according to the miRNA-gene assignment adjacency matrix. As each miRNA may be targeted to more than 100 genes, the potentially large size and complexity of a regulatory network containing more than 10 different miRNAs would make recognition of any miRNA-gene regulatory network difficult. The genes used in this miRNA-gene matrix were from miRNA profiles deemed significant. In these miRNA-gene networks, each gene and miRNA corresponded to a node and miRNA-gene interactions were connected by an edge. A miRNA-gene network was represented by adjacency matrix $\mathrm{A}=\left[a_{i j}\right]$, where $a_{i j}$ is the weight of the connection between gene "i" and miRNA "j". A definition for target hub genes with high density 3' UTR miRNAs was used. The centrality of the network was represented by a central degree. This describes the contribution thatgene " $\mathrm{i}$ " makes to the status (determining central status) of miRNA " $j$ " in the network or vice versa. The core gene or miRNA of a network is referred to as the main core or the highest degree of the graph and table.

Construction of gene ontology (GO)-miRNA regulatory network and topological analysis

Based on association of GO with genes, we can match GO terms to the genes that are targeted by miRNA with significant profiles. Correlation of miRNAs with GO terms could be defined through the gene name to construct the GO-miRNA adjacency matrix. Because each miRNA may be targeted to more than 100 genes with each gene assigned a few GO terms, the genes used in the miRNA-GO matrix correspondingly to significant miRNAs profiles. In a particular miRNA-GO network, each GO and miRNA corresponds to a node, and interactions between them are connected by an edge. Each miRNA-GO network can be represented by an adjacency matrix $\mathrm{A}=\left[a_{i j}\right]$, where $a_{i j}$ is the weight of a connection between GO "i" and miRNA "j". We also used a definition for target hub-GOs with particularly high density 3' UTRs miRNAs. The centrality of a network is represented by a central degree, which describes the contribution involved between the number of miRNAs targeting GO and the numbers of GO regulated by miRNAs. The core GO terms or miRNAs of the network are referred to as the highest degree of the graph.

\section{Real-time RT-PCR of miRNAs}

The differentially expressed miRNAs miR-133a and miR-155 were selected for verification.Expression of these mature miRNAs was assayed using stem-loop RT followed by PCR analysis according to the protocol from Applied Biosystems.PCR was performed in triplicate for each sample. The relative amount of miRNAs was normalized against miR-26b, and the fold change for each miRNA was calculated by the 2-[delta][delta] Ct method. The primers used for stem-loop RT-PCR were supplied by Applied Biosystems. 
Table 1. Levels of ALT, AST, STB, and LDH in mice serum

\begin{tabular}{lcccc}
\hline & ALT & AST & STB & LDH \\
& IU/L & IU/L & $\mu \mathrm{mol} / \mathrm{L}$ & IU/L \\
\hline Control group & $36.67 \pm 1.53$ & $85.33 \pm 4.04$ & $2.33 \pm 0.58$ & $1201.7 \pm 222.61$ \\
$1 \mathrm{hr}$ & $73 \pm 4.58$ & $149 \pm 15$ & $3.67 \pm 0.58$ & $946.67 \pm 158.09$ \\
$3 \mathrm{hr}$ & $137 \pm 8.89$ & $185.33 \pm 35.85$ & $5.33 \pm 0.58$ & $2784 \pm 942.13$ \\
$6 \mathrm{hr}$ & $1151.67 \pm 157.91$ & $1298.67 \pm 687.55$ & $6 \pm 1$ & $4059.67 \pm 2081.89$ \\
\hline
\end{tabular}

Table 2. Significant differentially expressed miRNAs and geometric mean of gene expression per time point (signal value)

\begin{tabular}{llllll}
\hline miRNA & $0 \mathrm{~h}$ & $1 \mathrm{~h}$ & $3 \mathrm{~h}$ & $6 \mathrm{~h}$ & $p$-value \\
\hline mmu-miR-133a & 10.6 & 14.1 & 15.5 & 701.3 & 0.0104378 \\
mmu-miR-182 & 40.9 & 19.7 & 17.8 & 30.2 & 0.0114823 \\
mmu-miR-203 & 1164.6 & 556.8 & 702.3 & 284.9 & 0.0177134 \\
mmu-miR-30e & 157.7 & 81.1 & 64 & 33.6 & 0.0346651 \\
mmu-miR-340-5p & 90.1 & 55.2 & 39.6 & 20.7 & 0.0435169 \\
mmu-miR-155 & 14.5 & 14.5 & 37.5 & 65 & 0.044013 \\
mmu-miR-377 & 35.2 & 17.2 & 18.8 & 38.4 & 0.0472745 \\
mmu-miR-805 & 2764.7 & 1398.1 & 2332.4 & 472.1 & 0.0518412 \\
mmu-miR-10a & 295.5 & 143.7 & 188.2 & 58.1 & 0.0537866 \\
mmu-miR-30a & 404.8 & 197 & 234 & 108.4 & 0.0588913 \\
mmu-miR-409-3p & 11.1 & 14.8 & 15.5 & 22 & 0.0821044 \\
mmu-miR-150 & 228.2 & 194.3 & 626.5 & 498.5 & 0.0853395 \\
\hline
\end{tabular}

Fig. 1. Liver haematoxylin and eosin staining. Balb/C mice received a ConA (20 $\mathrm{mg} / \mathrm{kg}$ ) injection via tail vein. One hour(b), 3 hours(c), and 6 hours(d) post-administration, livers were collected and stained with H\&E. Pyrogen-free normal saline was used as a negative control (a). Results are representative of four experiments (magnification, $\times 200$ ).

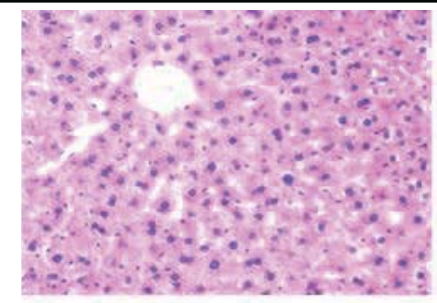

a

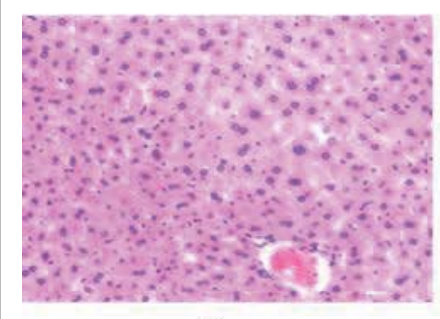

C

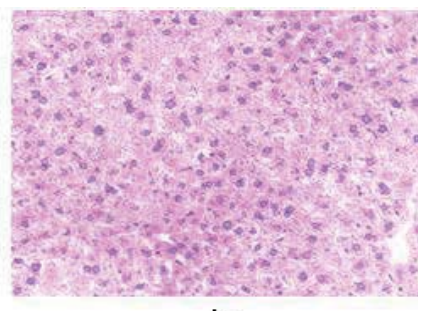

b

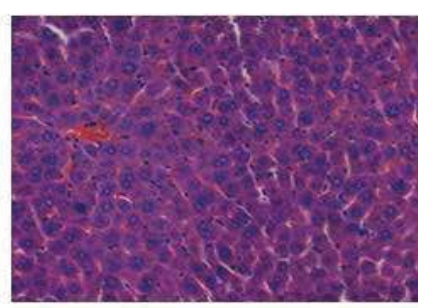

d

\section{Results}

ConA induces liver injury in BalB/c mice

Significant increases in serum alanine aminotransferase (ALT), aspartate aminotransferase (AST), lacticodehydrogenase (LDH), and total bilirubin (STB) associated with histological lesions were observed after ConA injection, as shown in Table 1 and Figure 1, indicating that severe acute hepatitis was successfully induced.

\section{Significant differential miRNAs among multiClass}

Randomized variance model correction of ANOVA was performed on samples from the four time points to determine those miRNAs that were truly separately and differentially expressed. The 11 miRNAs with a $p<0.1$ and FDR $<0.05$ were declared to be significantly 
Fig. 2. Expression profiles. The number in the top left hand corner of each profile box is the model profile ID number, and $p$-values are given in the lower left hand corner. Model expression profiles are ordered from left to right and top to bottom by increasing significance level. Dark profiles have statistical significance (corrected with multiple permutation test; $p<0.05$ ), and the difference in color has little meaning. Among the 26 profiles, 8 were confirmed profiles $(p<1)$, and only one profile (profile 3 ) showed significance $(p<0.05)$.
Profiles ordered based on the p-value significance of number of genes assigned versus expected
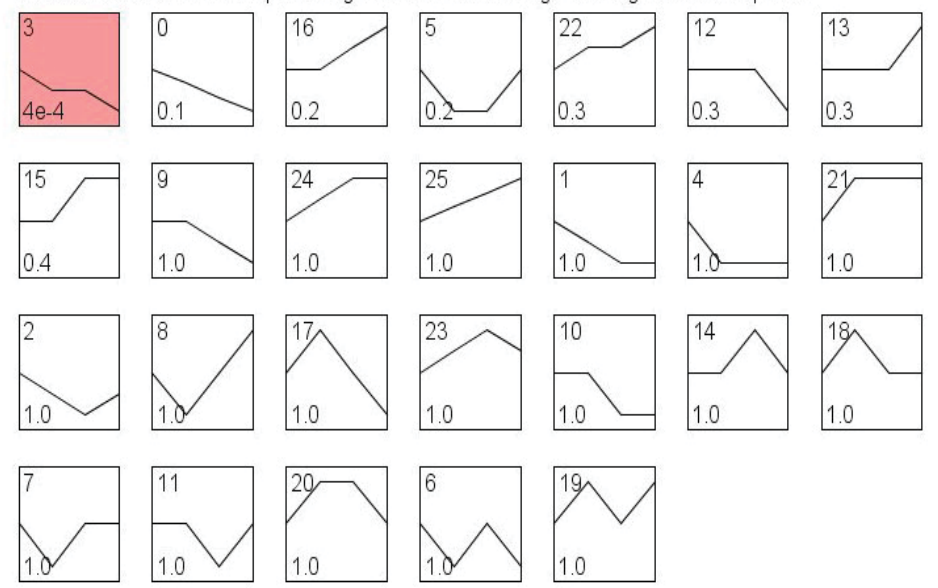

Fig. 3. Detailed expression profile 3. The horizontal axis represents the time points, and the vertical axis shows the time series of miRNA expression levels for the miRNA after log normalized transformation. Profile 3 at top center shows that the trend of the time series for the miRNA was $0,-1,-1,-2$. Assigned miRNA represents the number of miRNAs assigned to the profile. Expected miRNA represents the expected number of microRNAs based on a permutation test of time points. $p$-valuesindicate the significance of differences in the

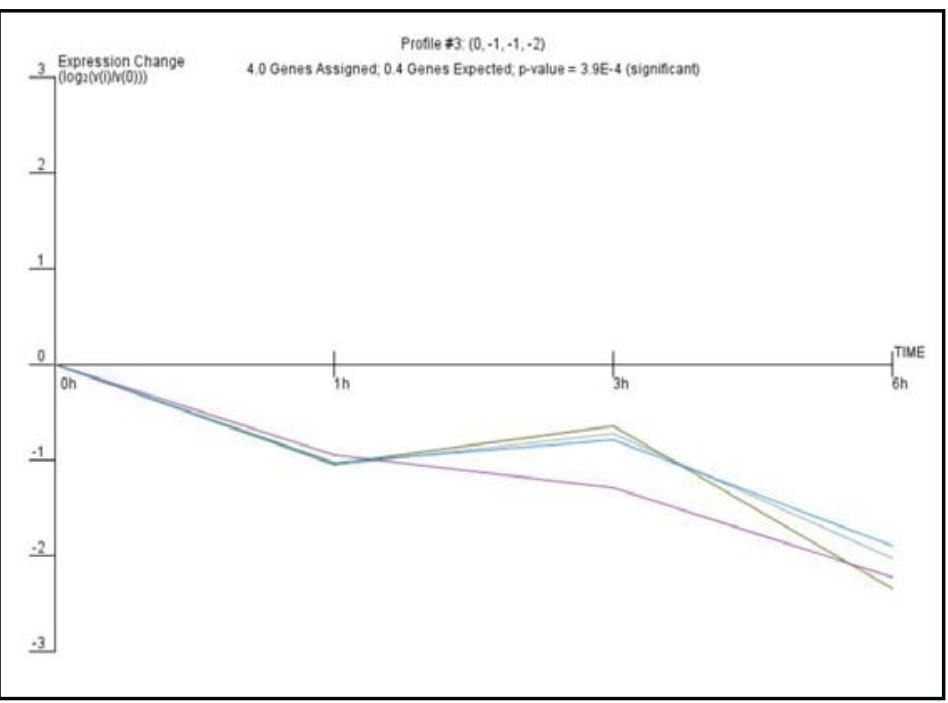
numbers of assigned versus expected miRNAs. Significance represents a change in the experiment versus the model profiles (the $p$-value is significant when a profile has more assigned than expected miRNAs).

Table 3. miRNA expression levels in miRNA profiles over time

\begin{tabular}{llllll}
\hline Profile & miRNA & $0 \mathrm{~h}$ & $1 \mathrm{~h}$ & $3 \mathrm{~h}$ & $6 \mathrm{~h}$ \\
\hline Profile 3 & $\mathrm{mmu}-\mathrm{miR}-203$ & 0 & -1.06 & -0.73 & -2.03 \\
Profile 3 & $\mathrm{mmu}-\mathrm{miR}-30 \mathrm{e}$ & 0 & -0.96 & -1.3 & -2.23 \\
Profile 3 & $\mathrm{mmu}-\mathrm{miR}-10 \mathrm{a}$ & 0 & -1.04 & -0.65 & -2.35 \\
Profile 3 & $\mathrm{mmu}-\mathrm{miR}-30 \mathrm{a}$ & 0 & -1.04 & -0.79 & -1.9 \\
\hline
\end{tabular}

differentially expressed. The value of the miRNA expression per group was calculated from the geometric mean of RMA normalization miRNA signals from three samples per time point. Detailed calculation of degree values are shown in Table 2.

Significant profiles of miRNA expression in liver injury induced by ConA

Eleven significantly differentially expressed miRNAs correlated by ANOVA and corrected by RVM were selected at the four time points, during the course of induced hepatitis. Twenty- 


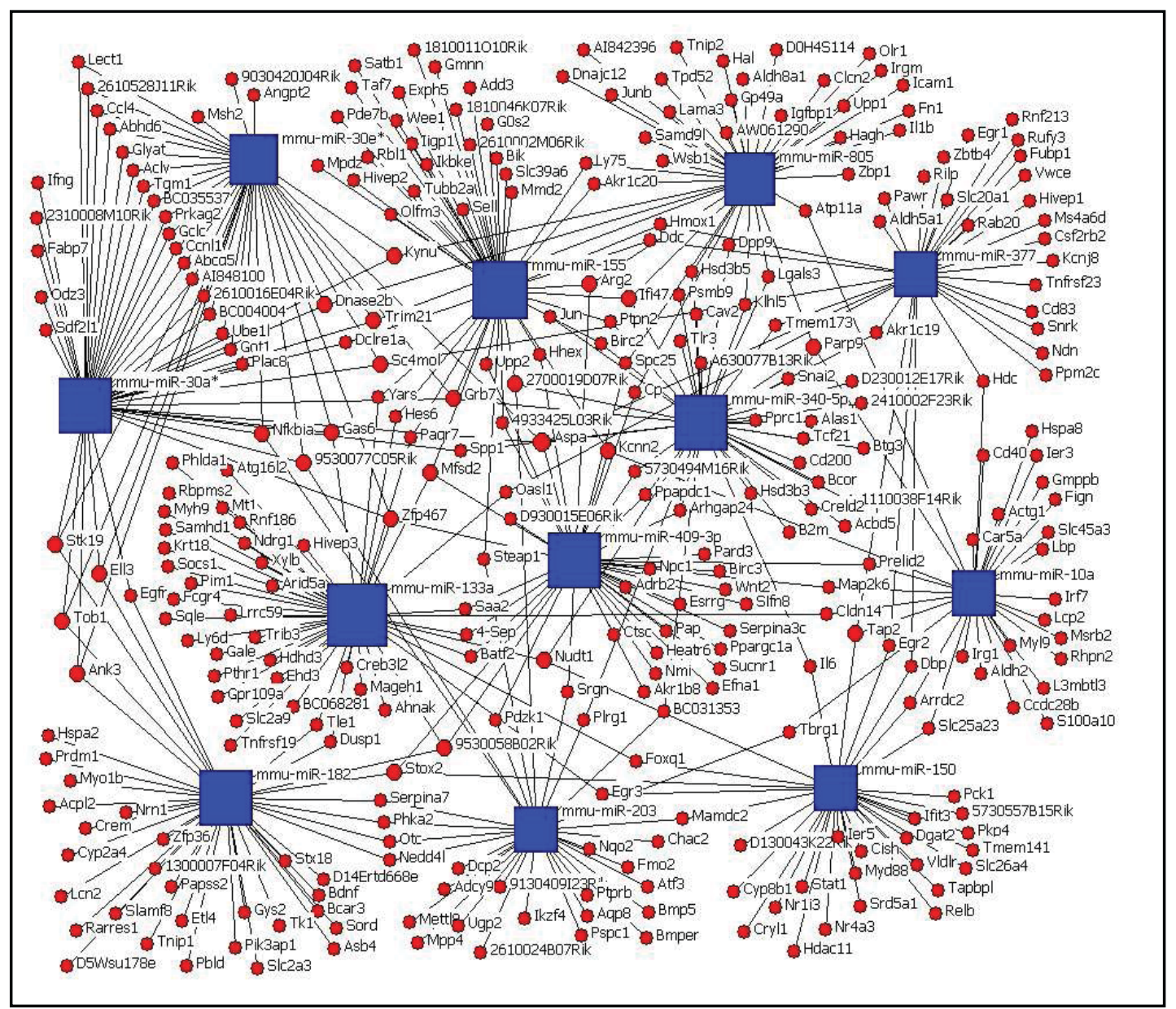

Fig. 4. miRNA-Gene-Network. Box nodes represent miRNA, and circle nodes represent mRNA. Edges describe the inhibition of mRNA by miRNA. The size of boxes represents the degree of miRNA ability to regulate mRNA.

six model profiles were detected, where the maximum unit change parameter was set to a value of 1(Fig. 2). Based on the assigned $p$ values, profile 3 was determined to be the most significant among these (Fig. 2, 3). If the pvalue of a profile was less than 0.05 , then the miRNA profile was deemed significant at that time point. Accordingly, the profile with the smallest $p$ value should have the highest correlation to regulatory miRNAs and the degree of their effect on liver tissue injury. Profile 3 has a significant level of $3.9 \times 10^{-4}$ and contained four microRNAs, which increased in expression with the duration of the injury induced after injecting ConA for $3 \mathrm{~h}$ (Table 3 ). This result indicates that the assigned miRNAs may closely correlate with the up- and down-regulation of miRNA expression during the course of injury caused by ConA.

\section{Core regulatory miRNAs and targeted hub genes}

After filtering significant miRNA profiles, miRNAs attributed to GO terms were defined as core miRNAs differentially controlling mRNAs. Then, miRNA-mRNA networks were constructed. After filtering of significant miRNA profiles, we identified separately two characteristics of the network: the relative ability of core regulatory miRNAs (boxes) to regulate mRNAs (boxes) (Fig. 4), and the relative levels of regulation of core target mRNAs (circles) to be regulated by the miRNAs (Fig. 5). We found that mmu-miR-133a was the most highly expressed, with strong regulatory ability, during the injury course and couldregulate 47 mRNAs (Table 4). This miRNA expression increased consistently over time: the geometric 


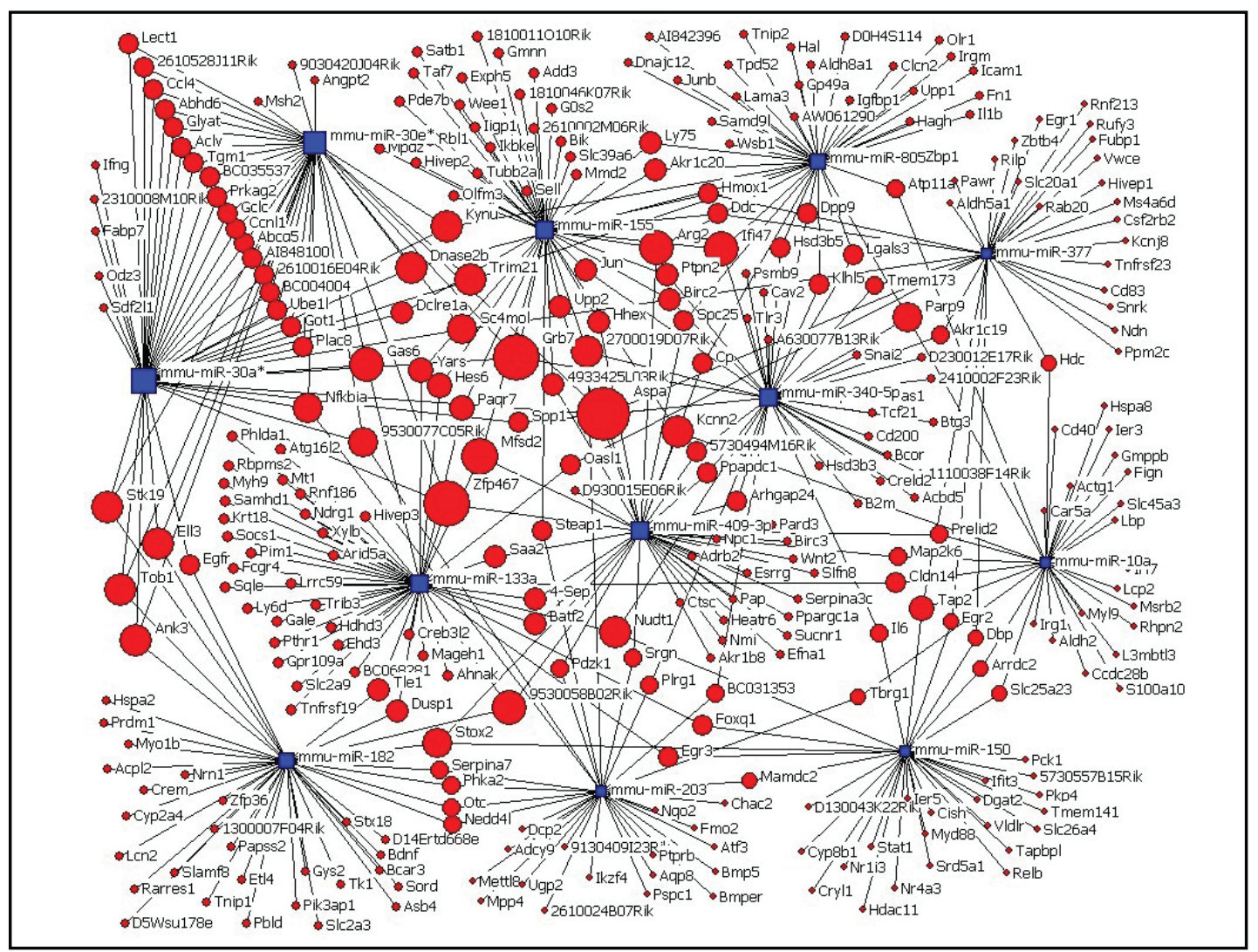

Fig. 5. miRNA-Gene-Network. Box nodes represent miRNA, and circle nodes represent mRNA. Edges describe the inhibition of mRNA by miRNA. The size of the circles represents the degree and status of mRNA control by miRNA.

Table 4. Degree of miRNA indicating the ability to regulate mRNA

\begin{tabular}{cc}
\hline miRNA & Degree \\
\hline $133 a$ & 47 \\
155 & 43 \\
182 & 40 \\
$30 a$ & 40 \\
$340-5 p$ & 40 \\
$409-3 p$ & 39 \\
805 & 38 \\
$30 \mathrm{e}$ & 35 \\
150 & 32 \\
203 & 31 \\
377 & 31 \\
$10 a$ & 30 \\
\hline
\end{tabular}

\begin{tabular}{lccccc}
\hline Gene & Degree & Gene & Degree & Gene & Degree \\
\hline Aspa & 5 & Dnase2b & 3 & Nudt1 & 3 \\
Grb7 & 4 & Ell3 & 3 & Parp9 & 3 \\
Zfp467 & 4 & Gas6 & 3 & Sc4mol & 3 \\
2700019D07Rik & 3 & Ifi47 & 3 & Stk19 & 3 \\
9530058B02Rik & 3 & Kcnn2 & 3 & Stox2 & 3 \\
9530077C05Rik & 3 & Kynu & 3 & Tap2 & 3 \\
Ank3 & 3 & Mfsd2 & 3 & Tob1 & 3 \\
Arg2 & 3 & Nfkbia & 3 & Trim21 & 3 \\
\hline
\end{tabular}

Table 5. Degree of miRNA control of mRNA expression

mean value of expression at the four time points $(0,1,3$, and $6 \mathrm{~h})$ were $10.6,14.1,15.5$, and 701.3, respectively. Additionally, the core target gene, Aspa, could be controlled by five miRNAs (Table 5). This gene was downregulated overtime, whereas expression of its single controlling miRNA, mmu-miR-409-3p, continuously increased. The geometric mean values of Aspa expression were 284, 209.4, 193.3, and 51 at the four time points, respectively. The geometric mean values of mmu-miR-409-3p were 11.1, 14.8, 15.5, and 22 at the four time points, respectively.

\section{Core regulatory miRNAs and targeted GO}

After filtration and selection of significant miRNA profiles, miRNAs attributed to GO terms were defined to be core miRNAs controlling differentially expressed mRNA groups. The miRNA-GO networks were then constructed (Fig. 6). The network nodes were defined 


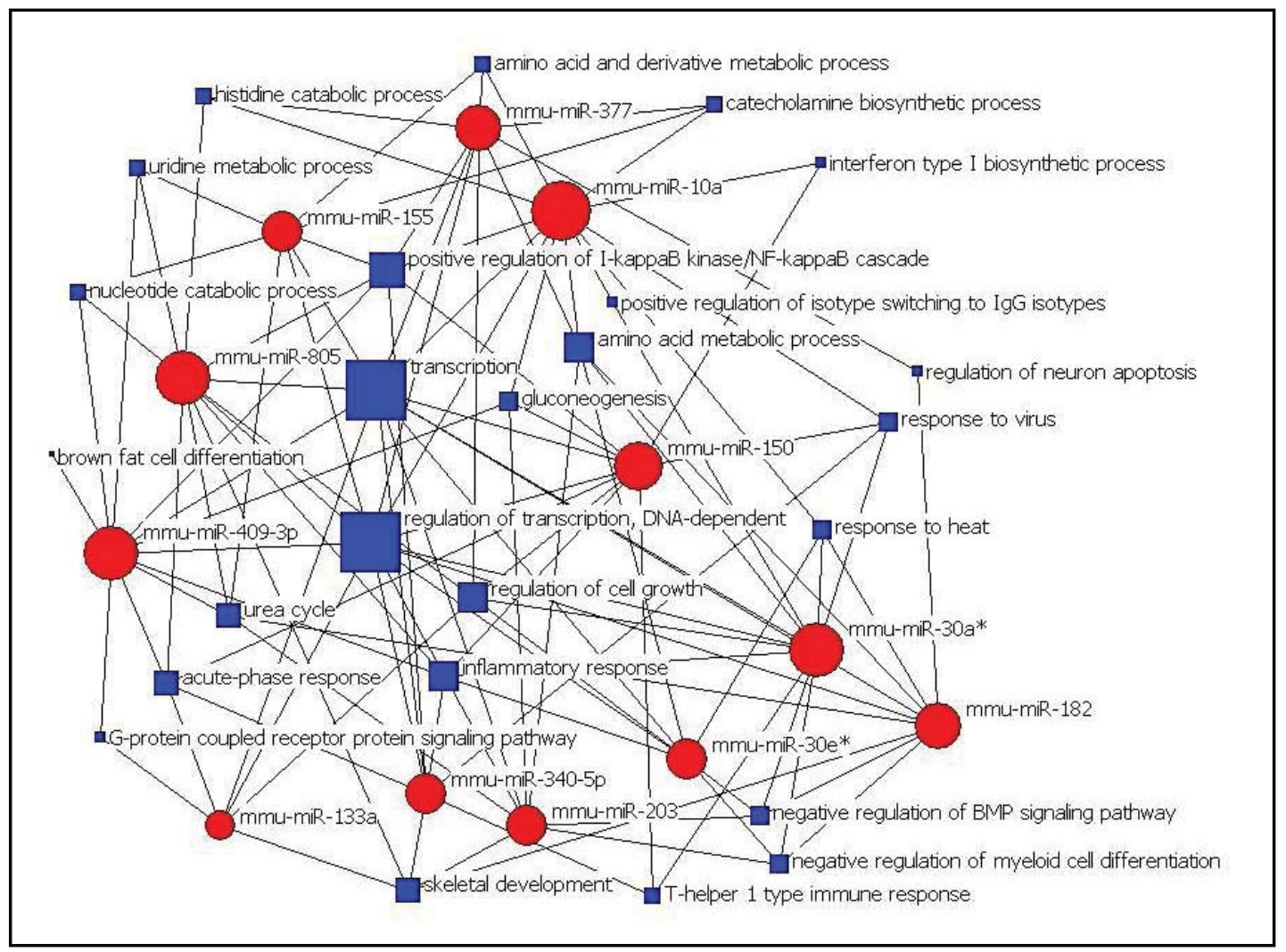

Fig. 6. miRNA-GO-Network. Box nodes represent GO, and circle nodes represent miRNA. Edges describe the inhibition of GO by miRNA. The size of the boxes represents the degree of miRNA ability to regulate GO.

by degree of regulation, and the highest degree GO terms or miRNAs were determined to be the most important nodes. The highest degree GO terms represented functions mainly controlled by miRNAs with a significant profile, whereas the highest degree miRNAs represented factors that may mainly regulate the functions of their target genes. We found that mmumiR-10a (Table 6) had the greatest ability during the course of injury to affect 12 cellular processes, of which transcription, DNA-dependent transcription, and the I-kappaB kinase/NF-kappaB pathway were most highly regulated(Fig. 6 and Table 7). In addition, mmu-miR-10a also inhibited the interferon type I biosynthetic process, response to heat, and response to virus, which may be associated with inflammation induced by a viral infection.

The three top degree GOs (Table 7), regulation of transcription, DNAdependent transcription, and positive regulation of I-kappaB kinase/ $N F$-kappaB were the three top gene functions most targeted by miRNAs,
Table 6. Degree of miRNA ability to regulate GO

\begin{tabular}{cc}
\hline miRNA & Degree \\
\hline $10 \mathrm{a}$ & 12 \\
$30 \mathrm{a}$ & 11 \\
$409-3 \mathrm{p}$ & 11 \\
805 & 11 \\
150 & 10 \\
182 & 9 \\
377 & 9 \\
155 & 8 \\
203 & 8 \\
$30 \mathrm{e}$ & 8 \\
$340-5 \mathrm{p}$ & 8 \\
$133 \mathrm{a}$ & 6 \\
\hline
\end{tabular}
which could be inhibited by ConA. As a consequence, response to heat and the response to viral inflammation could be suppressed. In addition, the aminoacid and derivative metabolic process, catecholamine biosynthetic process, gluconeogenesis, and the histidine catabolic process involved in liver metabolism could also be inhibited (Fig. 6). All the above-mentioned processes were inhibited by mmu-miR-10a.

\section{Real-time RT-PCR of miRNAs}

Two differentially expressed miRNAs, miR-133a and miR-155,were selected for verification.The relative amounts of miRNAs normalized against miR-26b are listed in Figure 7. 
Table 7. Degree of GO control by miRNA

\begin{tabular}{ll}
\hline GO & Degree \\
\hline Regulation of transcription, DNA-dependent & 12 \\
Transcription & 12 \\
Positive regulation of I-kappaB kinase/NF-kappaB cascade & 7 \\
Amino-acid metabolic process & 6 \\
Inflammatory response & 6 \\
Regulation of cell growth & 6 \\
Acute-phase response & 5 \\
Skeletal development & 5 \\
Urea cycle & 5 \\
Gluconeogenesis & 4 \\
Negative regulation of BMP signaling pathway & 4 \\
Negative regulation of myeloid cell differentiation & 4 \\
Response to heat & 4 \\
Response to virus & 4 \\
Amino acid and derivative metabolic process & 3 \\
Catecholamine biosynthetic process & 3 \\
Histidine catabolic process & 3 \\
Nucleotide catabolic process & 3 \\
T-helper 1 type immune response & 3 \\
Uridine metabolic process & 3 \\
G-protein coupled receptor protein signaling pathway & 2 \\
Interferon type I biosynthetic process & 2 \\
Positive regulation of isotype switching to IgGisotypes & 2 \\
Regulation of neuron apoptosis & 2 \\
Brown fat cell differentiation & 1 \\
\hline
\end{tabular}

Fig. 7. The fold changes in miR133a and miR-155 expression. Differentially expressed miRNAs miR133a and miR-155 were selected for verification. The relative amount of miRNAs was normalized against miR-26b, and the fold change for each miRNA was calculated by the 2-[delta][delta] Ct method.

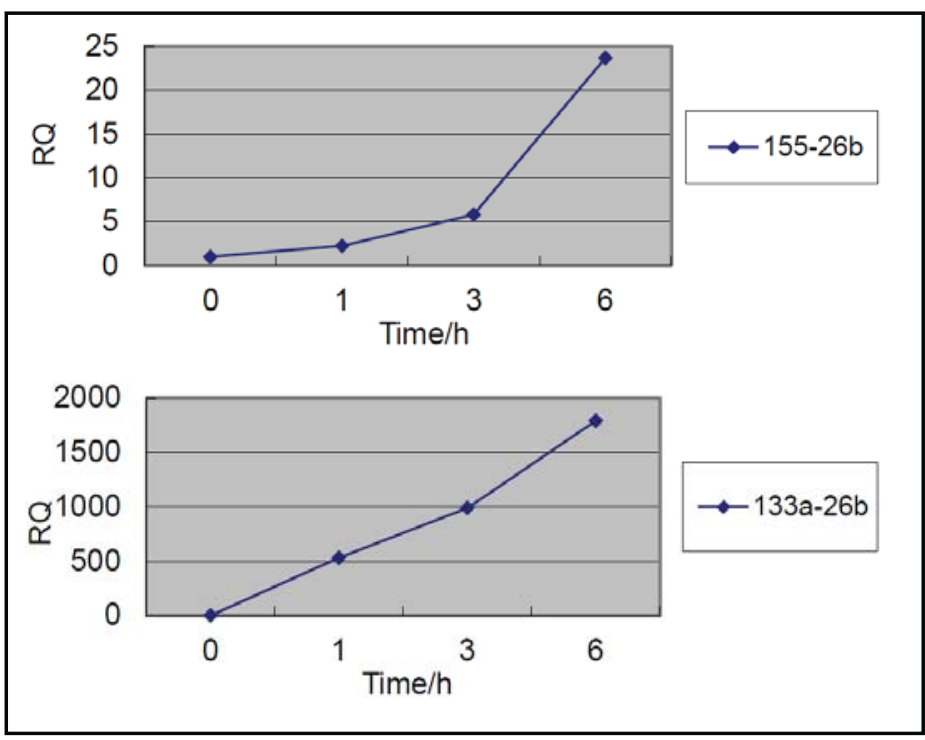

\section{Discussion}

In this study, mice were injected with ConA to induce severe acute hepatitis as confirmed by biochemical and histological data.T hese features, together with mortality, increased over time, reaching a peak at $24 \mathrm{~h}$. It has been previously reported that only mild lung congestion and mild renal tubular epithelial cell vacuolar degeneration are found in mice given ConA without heart or brain damage, suggesting that severe liver damage was the cause of death $[11,12]$.

The purpose of this study was to investigate miRNA expression profiles in murine ConA-induced liver damage up to $6 \mathrm{~h}$, combined with gene expression profile analyses, to 


\section{Cellular Physiology and Biochemistry}

Cell Physiol Biochem 2014;33:1933-1944

DOI: $10.1159 / 000362970$

Publisned online: June 27, 2014

C) 2014 S. Karger AG, Basel

www.karger.com/cpb

Jia et al.: ConA-Induced Hepatitis MicroRNA Expression Profiles

determine miRNA involvement in the regulation and exacerbation of liver inflammation. miRNAs are known to regulate many aspects of the innate and acquired immune responses, including inflammation [13,14]. However, the miRNAs involved in fulminant hepatitis have not yet been extensively investigated. We predefined special sets of profiles according to those expected following stimulation with ConA. However, due to the noise and the small number of points for each miRNA, the profiles are shared by many miRNAs and are expected to appear by chance. We first selected a set of potential expression profiles. These 26 sets of profiles covered the number of possible profiles that could be regulated by miRNA expression in the course of stimulation with ConA at four time points, and each represents a single temporal expression profile. Enrichment of miRNA profiles was used to measure the significance of each profile. Profiles reflecting common functions attributed to miRNA co-expression and functions that influence related biological processes were considered significant. In this study, the designated profile 3 was highly statistically significant.

In the post-transcription process, miRNAs can regulate gene expression through mRNA degradation and mRNA translation inhibition. The function of a biological system can be changed by miRNA over expression. One miRNA can inhibit multiple genes or GOs, and one gene or GO can be inhibited by multiple miRNAs. As a result, a network represents the relationship between miRNAs, genes, and GOs.

Because of the flexibility of the network model based on the algorithms predicted from high throughput miRNA expression tests, we can look at snapshots of protein-protein interactions, miRNA-gene expression regulatory networks, miRNA-GO expression regulatory networks, and metabolism networks among different groups. The intrinsic miRNA-gene network of a particular phenotype can represent miRNA function. A miRNA-GO network could provide a more in-depth analysis and visualization of miRNAs' effects. The degrees of miRNAs, gene, and GO interactions are key attributes in a network. In this study, we used the degrees of miRNA-gene and miRNA-GO interaction networks to define main miRNAs, mRNAs, or GOs as central nodes in a main network. The rank of degree value describes the complexity of miRNA associations. Conversely, the complexity of the miRNA relationship increases with the degree value rank.

In conclusion, we found that two miRNAs, miR-133a and miR-155, were highly expressed, with strong regulatory ability during the course of injury (Fig. 4, 5 and Table 3). Forty-seven genes were found to be regulated by miR-133a, and 43 genes by miR-155. miR133a may regulate skeletal muscle proliferation and differentiation [15] and could be a key regulator of cardiac hypertrophy [16]. Previous reports revealed that miR-155 was thought to regulate the acute inflammatory response and is also implicated in $\mathrm{B}$ - and $\mathrm{T}$-cell responses [17-19]. Furthermore, An et al. reported that miR-155 is up-regulated at both 5 and $7 \mathrm{~h}$ post-challenge in D-galactosamine-induced ALF [8]. Other miRNAs that were ranked with a high degree of regulatory ability in this study included: miR-182, miR-30a*, miR-340-5p, miR-409-3p, miR-805, miR-30e*, miR-150, miR-203, miR-377, and miR-10a. Among these miRNAs, miR-150 controls B-cell differentiation by targeting the transcription factor $\mathrm{c}-\mathrm{Myb}$ [20], and miR-150 and miR-186 down-regulate expression of the pro-apoptotic purinergic P2X7 receptor. This down regulation results from the activation of instability sites at the 3'UTR of the gene, which decreases steady-state levels of the transcript [21]. Future studies using the murine ConA-induced hepatitis model are warranted to obtain further insight into the functional understanding of miRNAs.

\section{Acknowledgments}

This work was supported by National Natural Science Foundation of China (81201291), National Science and Technology Major Project (2012ZX10002003), Science and Technology Major Projects of Zhejiang Province (2009C03011-2). We thank Medjaden Bioscience Limited for assisting in the preparation of this manuscript. 


\section{Cellular Physiology and Biochemistry}

Cell Physiol Biochem 2014;33:1933-1944

\begin{tabular}{l|l}
\hline DOI: $10.1159 / 000362970$ & (C) 2014 S. Karger AG, Basel
\end{tabular}

www.karger.com/cpb

Jia et al.: ConA-Induced Hepatitis MicroRNA Expression Profiles

\section{References}

1 Matsumoto G, Tsunematsu S, Tsukinoki K, Ohmi Y, Iwamiya M, Oliveira-dos-Santos A, Tone D, Shindo J, Penninger JM: Essential role of the adhesion receptor lfa- 1 for $t$ cell-dependent fulminant hepatitis. J Immunol 2002;169:7087-7096.

2 Nicoletti F, Beltrami B, Raschi E, Di Marco R, Magro G, Grasso S, Bendtzen K, Fiorelli G, Meroni PL: Protection from concanavalin a (con a)-induced t cell-dependent hepatic lesions and modulation of cytokine release in mice by sodium fusidate. Clin Exp Immunol 1997;110:479-484.

-3 Liu LL, Gong LK, Wang H, Xiao Y, Wu XF, Zhang YH, Xue X, Qi XM, Ren J: Baicalin protects mouse from concanavalin a-induced liver injury through inhibition of cytokine production and hepatocyte apoptosis. Liver Int 2007;27:582-591.

4 Bartel DP, Chen CZ: Micromanagers of gene expression: The potentially widespread influence of metazoan micrornas. Nat Rev Genet 2004;5:396-400.

5 Lindsay MA: Micrornas and the immune response. Trends Immunol 2008;29:343-351.

6 Sharma AD, Narain N, Handel EM, Iken M, Singhal N, Cathomen T, Manns MP, Scholer HR, Ott M, Cantz T: Microrna-221 regulates fas-induced fulminant liver failure. Hepatology 2011;53:1651-1661.

7 Xi D, Wang M, Ye H, Luo X, Ning Q: Combined adenovirus-mediated artificial micrornas targeting mfgl2, mfas, and mtnfr1 protect against fulminant hepatic failure in mice. PLoS One 2013;8:e82330.

-8 An F, Gong B, Wang H, Yu D, Zhao G, Lin L, Tang W, Yu H, Bao S, Xie Q: Mir-15b and mir-16 regulate tnf mediated hepatocyte apoptosis via bcl2 in acute liver failure. Apoptosis 2012;17:702-716.

-9 Chen F, Zhu HH, Zhou LF, Li J, Zhao LY, Wu SS, Wang J, Liu W, Chen Z: Genes related to the very early stage of cona-induced fulminant hepatitis: A gene-chip-based study in a mouse model. BMC Genomics2010;11:240.

-10 Stupar RM, Gardiner JM, Oldre AG, Haun WJ, Chandler VL, Springer NM: Gene expression analyses in maize inbreds and hybrids with varying levels of heterosis. BMC Plant Biol 2008;8:33.

-11 Bogdan C, Mattner J, Schleicher U: The role of type i interferons in non-viral infections. Immunol Rev 2004;202:33-48.

12 Roda JM, Parihar R, Carson WE, 3rd: Cpg-containing oligodeoxynucleotides act through tlr9 to enhance the nk cell cytokine response to antibody-coated tumor cells. J Immunol 2005;175:1619-1627.

13 Urbich C, Kuehbacher A, Dimmeler S: Role of micrornas in vascular diseases, inflammation, and angiogenesis. Cardiovasc Res 2008;79:581-588.

14 Sonkoly E, Stahle M, Pivarcsi A: Micrornas and immunity: Novel players in the regulation of normal immune function and inflammation. Semin Cancer Biol 2008;18:131-140.

15 Chen JF, Mandel EM, Thomson JM, Wu Q, Callis TE, Hammond SM, Conlon FL, Wang DZ: The role of microrna-1 and microrna-133 in skeletal muscle proliferation and differentiation. Nat Genet 2006;38:228233.

16 Care A, Catalucci D, Felicetti F, Bonci D, Addario A, Gallo P, Bang ML, Segnalini P, Gu Y, Dalton ND, Elia L, Latronico MV, Hoydal M, Autore C, Russo MA, Dorn GW, 2nd, Ellingsen O, Ruiz-Lozano P, Peterson KL, Croce CM, Peschle C, Condorelli G: Microrna-133 controls cardiac hypertrophy. Nat Med 2007;13:613-618.

-17 O'Connell RM, Taganov KD, Boldin MP, Cheng G, Baltimore D: Microrna-155 is induced during the macrophage inflammatory response. Proc Natl Acad Sci U S A 2007;104:1604-1609.

18 Tili E, Michaille JJ, Cimino A, Costinean S, Dumitru CD, Adair B, Fabbri M, Alder H, Liu CG, Calin GA, Croce CM: Modulation of mir-155 and mir-125b levels following lipopolysaccharide/tnf-alpha stimulation and their possible roles in regulating the response to endotoxin shock. J Immunol 2007;179:5082-5089.

19 Rodriguez A, Vigorito E, Clare S, Warren MV, Couttet P, Soond DR, van Dongen S, Grocock RJ, Das PP, Miska EA, Vetrie D, Okkenhaug K, Enright AJ, Dougan G, Turner M, Bradley A: Requirement of bic/microrna-155 for normal immune function. Science 2007;316:608-611.

20 Xiao C, Calado DP, Galler G, Thai TH, Patterson HC, Wang J, Rajewsky N, Bender TP, Rajewsky K: Mir-150 controls b cell differentiation by targeting the transcription factor c-myb. Cell 2007;131:146-159.

-21 Zhou L, Qi X, Potashkin JA, Abdul-Karim FW, Gorodeski GI: Micrornas mir-186 and mir-150 downregulate expression of the pro-apoptotic purinergic $\mathrm{p} 2 \mathrm{x} 7$ receptor by activation of instability sites at the 3 '-untranslated region of the gene that decrease steady-state levels of the transcript. J Biol Chem 2008;283:28274-28286. 